\title{
Anthropomorphic Language in Online Forums about Roomba, AIBO and the iPad
}

\author{
J. Fink, O. Mubin, F. Kaplan, P. Dillenbourg
}

\begin{abstract}
What encourages people to refer to a robot as if it was a living being? Is it because of the robot's humanoid or animal-like shape, its movements or rather the kind of interaction it enables? We aim to investigate robots' characteristics that lead people to anthropomorphize it by comparing different kinds of robotic devices and contrasting it to an interactive technology. We addressed this question by comparing anthropomorphic language in online forums about the Roomba robotic vacuum cleaner, the AIBO robotic dog, and the iPad tablet computer. A content analysis of 750 postings was carried out. We expected to find the highest amount of anthropomorphism in the AIBO forum but were not sure about how far people referred to Roomba or the iPad as a lifelike artifact. Findings suggest that people anthropomorphize their robotic dog significantly more than their Roomba or iPad, across different topics of forum posts. Further, the topic of the post had a significant impact on anthropomorphic language.
\end{abstract}

\section{INTRODUCTION}

$\mathrm{T}$ HE phenomenon of anthropomorphism - in contrast to anthropomorphic form in design - describes people's tendency to attribute human-/lifelike qualities (e.g. intentions, motivations, emotions, characteristics) to non-lifelike objects [1] [2] and is described as a social response to technology. Though there are specific understandings of humanor animal-likeness and anthropomorphism or zoomorphism, we do not distinguish them here. The trend of anthropomorphism has been widely explored with emerging new interactive technologies, in human-computer interaction [3] as well as in respect of technology acceptance [4]. Discussions about anthropomorphism have recently become popular also in matters of the design of (social) robots [5] [6] [7]. Arising questions are how far robots are perceived as moral or social agents and how far they should resemble human shape and/or qualities at all. Philosophical and ethical issues are not addressed in this paper but can be found in [2] [8], for instance. Nevertheless, the understanding of how and why people anthropomorphize their technology / robot is of interest when it comes to designing future devices that are in close contact to humans, such as domestic robots. An interesting question here is how to design robots that are likely to elicit social

Manuscript received December 20, 2011. This research was supported by the Swiss National Science Foundation through the National Centre of Competence in Research on Robotics.

J. Fink, O. Mubin, F. Kaplan, P. Dillenbourg are with CRAFT, Ecole Polytechnique Fédérale de Lausanne (EPFL), CH-1015 Lausanne, Switzerland (phone: +41 (0) 2169320 61; fax: +41 (0) 2169360 70; e-mail: julia.fink; omar.mubin; frederic.kaplan; pierre.dillenbourg @epfl.ch). responses from their users which in turn forms people's expectations of the robot and could also enhance acceptance, for instance [4] [5] [9]. Epley et al. argue that anthropomorphized agents can act as powerful agents of social connection. Further, anthropomorphizing technological agents appears to aid in effectively learning how to use those agents. Accordingly, previous work suggests that how social a system or product appears, positively affects how pleasant and usable it is perceived [10] and how far people feel empathetic towards it [11]. Further, it has been reported that people are likely to spend more effort in learning how to use the device [1] and preferred to collaborate with a robot that was able to respond socially (e.g. by displaying different facial expressions) [9] [12]. Besides psychological determinants [1] that advantage people's usage of anthropomorphic language, we aim to explore factors originating from the artifact that facilitate anthropomorphizing, such as interaction modalities or the physical shape of the device. We believe that an understanding of how and why people anthropomorphize a robot could help us to identify the particular characteristics that make the device likely to be perceived lifelike and to relate socially to it.

We assumed that a robot's ability to move and respond to the user in an individualized way could be one of the cues that might lead people to anthropomorphize it. We investigated this through a content analysis of online discussion forums comparing postings about AIBO, Roomba and the iPad (Fig. 1).
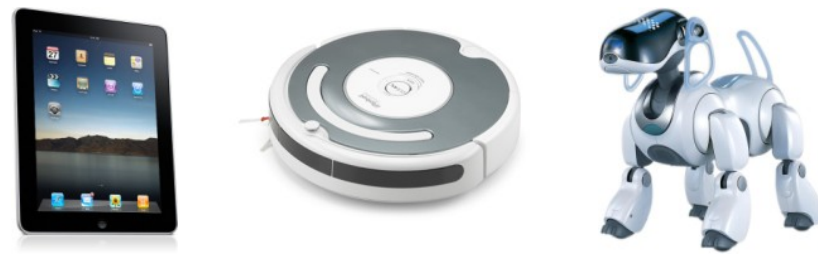

Fig. 1 Tablet computer iPad, vacuum cleaning robot Roomba and robotic $\operatorname{dog}$ AIBO.

We identified anthropomorphic language in the forum posts as well as the context in which it occurred. Particularly, we were interested to see where those three devices range on a subjective scale of anthropomorphism. For this, we need to ask first how these three devices differ from one another and how their specific characteristics can possibly encourage anthropomorphizing them. We take it as an assumption that how we talk or write about an artifact reveals something about how we relate to it. Though linguistics also implicates 
a cognitive process, we will not address this here. Related psychological factors explaining the phenomenon of why people tend to anthropomorphize can be read in [1]; a study about people's mental models of robots can be found in [13]. In the following we review related work on anthropomorphism in robotics, describe our own study, present findings, and will discuss factors that seem to encourage people to treat an artifact as if it was lifelike.

\section{RELATED WORK}

There are two main perspectives when seeking to explain people's tendency to anthropomorphize artifacts [14]: a rather artifact-centered and a person-centered approach.

First one explains anthropomorphism from the design of the artifact. It is assumed that humans directly respond to lifelike or social cues that an object or system emits. Up to a certain degree, anthropomorphic form creates familiarity and increases the likelihood of relating socially to the artifact. Encouraging anthropomorphism through the robot's design (embodiment as well as behavior) can serve as a method to increase the feeling of being socially connected to the robot and its perceived usefulness [1]. For example, people responded more positively to an artifact that displayed humanlike behavioral characteristics (emotions, facial expression) in contrast to a purely functional design [3] [6] [11] [15]. However, user preferences were task and context dependent, indicating that people preferred a more humanlike robot for jobs requiring more sociability [12].

A second explanation applies a human-centered, cognitive viewpoint where anthropomorphism is described through people's specific mental model they have about how an artifact works the way it does. The explanation builds on social theories of human-human communication [13]. When communicating with other people, we make assumptions about what those people know and adjust our language accordingly. This can for example be observed when talking to young children. Besides other factors, the estimations and expectations we have about others determine the way we talk (or interact) with them. Similarly, people's estimation of a robot's "knowledge" and its capabilities/abilities is said to affect the way we relate to it. Studies examined the validity of the mental model concept with various kinds of robots [13] [14]. Findings suggest that people tend to hold richer mental models about anthropomorphic robots in contrast to mechanic ones and also feel more confidence in rating them according to several personality traits [13].

As an alternative to these two explanations, one can explain people's tendency to attribute human qualities to objects based on social psychology. Epley et al. established a three-factor theory of when people are likely to anthropomorphize based on psychological determinants and rather independent from artifact-based characteristics or mental models. Namely, the theory describes that some people are more likely to anthropomorphize, so when (i) anthropocentric knowledge is accessible and applicable to the artifact (elicited agent knowledge), (ii) they are motivated to explain and understand the behavior of other agents (effectance motivation), and (iii) they have the desire for social contact and affiliation (social motivation) [1]. In other words, (i) describes people's tendency to use knowledge about humans (or self-knowledge) as a basis for explaining nonhuman agents. The second factor, (ii) refers to human's need of interacting effectively with one's environment, e.g. by being able to explain complex stimuli in the present and predict the behavior of these stimuli in the future. With the author's words: "Attributing human characteristics and motivations to nonhuman agents increases the ability to make sense of an agent's actions, reduces the uncertainty associated with an agent, and increases confidence in predictions of this agent in the future." [Epley et al, p. 866] As a third (iii) key psychological determinant for anthropomorphism, the authors mention a social motivation: In the absence of social connection to other humans people tend to create human agents out of nonhumans through anthropomorphism in order to satisfy their motivation for social connection.

Originating from the notion of anthropomorphism, research aimed to investigate characteristics of social relationships between humans and robots. Bartneck et al. suggest several measurement instruments, amongst others for anthropomorphism [16]. Friedman et al. [17] analyzed online forum posts, in order to find out how far people describe their robotic devices as companions. Assumed that the social relationship to the robot is reflected by people's language use, they examined written discussions about the robotic dog AIBO by means of a content analysis. Results showed that besides referring to AIBO in terms of life-like essences (it has "eyes", "ears", etc.), or comparing the robotic pet directly to a biological dog, people talked about AIBO as if it had mental states (having intentions or feelings), as if it were a social companion (talking directly to it, considering it as a family member) or even a moral agent [17]. Our study builds on these results but goes beyond by comparing the use of anthropomorphisms between different technologies, with or without robotic components. Further, we investigate whether the topic of the conversation impacts the use of anthropomorphic notions. We believe that the context of an interaction is an important factor for a holistic understanding of anthropomorphism. Results can serve to obtain design suggestions for robots that encourage social engagement.

\section{STUdY DESIGN AND Methodology}

Similar to the approach applied by Friedman et al. [17], we studied anthropomorphic language in online forums. People registered as authors in one of the forums write about their experiences with the device, how they use it, or ask others for help when encountering technical problems, for instance. However, our work goes beyond previous work by comparing the amount and context of anthropomorphic language for three different interactive devices. This comparison enables us to obtain a subjective scale of how far the 
three devices encourage people to anthropomorphize it and further helps to identify concrete characteristics for designing products that facilitate social connection when desired. This goal further led us to choose on the one hand a pet robot and a functional domestic service robot, and on the other hand a multi-touch display tablet computer. We assumed that the different shape as well as the functionality and interaction that each of these three products enable will encourage people accordingly to anthropomorphize the object or not.

Based on previous work, we hypothesized that people engage in a more social way with a robotic pet (AIBO) compared to a functional robot (Roomba) or a tablet computer (iPad), and thus expected more anthropomorphic language in the AIBO forum $\left(\mathrm{H}_{1}\right)$. Since anthropomorphism displays a certain kind of emotional / social relation to the artifact, we further hypothesized anthropomorphic language would be more likely to occur in forum posts where the author describes her social relation to the device in comparison to discussing technological aspects or how the device is used $\left(\mathrm{H}_{2}\right)$.

\section{A. Coding for the Topic of the Posts}

We verified our assumptions through a content analysis, comparing the amount and the context of anthropomorphic language in online forums about AIBO (www.aibo-life.org), Roomba (www.robotreviews.com), and the iPad (www.ipadforums.net). Selection criteria for the forums were the language (English), the community size (> 50 authors), and being up-to-date (ten latest posts not older than six months). 250 posts of each forum have been quasi-randomly selected. This means that we did not extract too many posts from one single conversation but aimed to cope with the range of topics of the respective forum. The extracted posts were further split into segments to allow precise coding. Segmentation was done according to paragraphs in the written text. This was necessary, as some conversations have been quite long and could not be coded univocally.

After this process, out from the 750 posts, $n=1363$ distinct segments were obtained. A robust coding scheme has been established with two dependent variables: content (the topic of the segment) and anthropomorphism. First, the topic of each segment was annotated in three categories:

-- "technology" for technological aspects, a functional problem or broken parts;

-- "usage" when the segment described how or for what people use their device (an activity); and

-- "relationship" when an attitude or feeling towards the device was described.

The additional category "irrelevant" was used to filter out completely unrelated posts. From the 1363 coded segments, 155 were unrelated. Further statistical analysis has been carried out only with the remaining 1208 segments.

\section{B. Coding for Anthropomorphic Language}

In a second step, when a forum segment was not previously coded as irrelevant, it was labeled as anthropomorphic or not. It took several approaches to operationalize when a post should be coded as "anthropomorphic". We finally defined several categories of an anthropomorphism (adapted also from [17]). A segment was coded as anthropomorphic when the device was described in terms of:

-- life-likeness, such as being alive or having (parts of) a body (e.g. "she can be reborn since you copied her memory stick");

-- emotional states or having a feeling (e.g. "oh no, poor AIBO");

-- gender, personality or having an intention (e.g. Roomba "seems to like to hang around" under the sofa);

-- when the author gave it a name (e.g. "whenever I show Java his pink ball");

-- socially integrated, such as a family member (e.g. "I' $m$ considering adding a Roomba to the family");

-- metaphorical ways (e.g. Roomba "sings its victory song when it finishes and docks.").

Each segment was coded in only one content category and either as "anthropomorphic" or "not anthropomorphic".

To assure the validity of our coding scheme, a second coder randomly annotated $20 \%$ of the segments equally for each device. Cohen's Kappa was 0.78 for anthropomorphism and 0.6 for content which indicates a moderate to substantial inter-rater reliability. Disagreements for content were due to confusion between the labels "technology" and "usage". The disagreements were resolved through discussion.

\section{FINDINGS}

With respect to the three different topics, in total, most conversations concerned a technical aspect $(67.7 \%)$ in comparison to descriptions of how people used their device $(21.8 \%)$ and how they felt about it $(10.5 \%)$. Especially in the Roomba forum, technical questions and answers $(79.9 \%)$ dominated the topics usage $(15.9 \%)$ and relation (4.2\%) (Fig. 2).

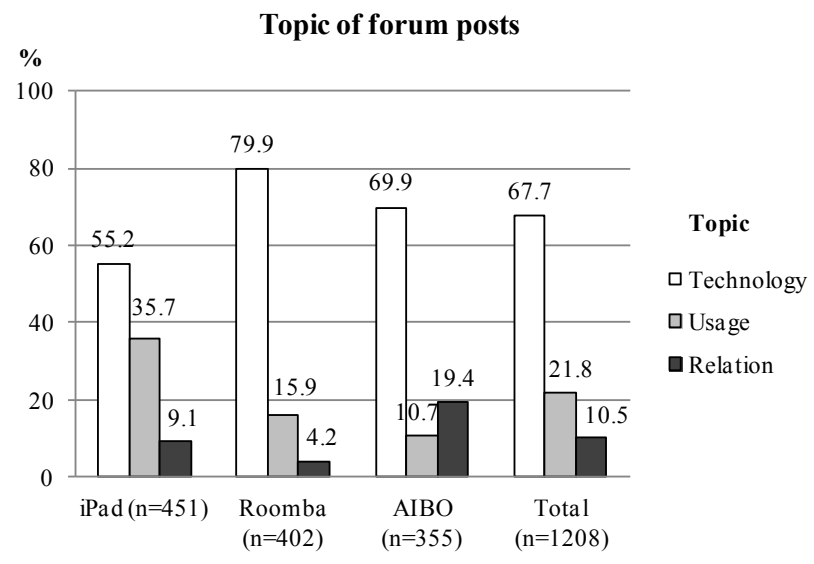

Fig. 2. Topic of forum posts comparing three devices and in total 
Roomba owners frequently described their vacuum cleaner was not able to properly navigate autonomously around the home or would not get back to its recharging station. It was often mentioned the robot reported errors or stopped working, so people sought for help explaining how their Roomba showed defective behavior while vacuuming. Further, people tended to write in the forum right after they had purchased a Roomba and asked others about how the robot worked:

"How does the Roomba know the room is clean? If $i$ put it in a bedroom and push the clean button, close the door to confine it, how long will it run? Does it go until the bateries are dead? " [original text]

Across the three different topic categories the amount of anthropomorphic language in the Roomba forum varied in between $9.7 \%$ and $23.5 \%$, of where the highest proportion was found in the relation topic (Fig. 3).

The main part of conversations in the iPad forum (55.2\%) dealt with technical concerns, such as compatibility problems with iTunes and a PC system. Further, about one Third (35.7 $\%$ ) of the postings described use cases, where and for what people used their iPad and how far it was able to replace a laptop or notebook (Fig. 2). The remaining 9.1\% of the conversations have been about how people felt about their iPad, whether they liked it or hated it and for which reasons:

"This is my first iPad [...]. Personally, I love it. I like the more restricted and uniformed work flow compared to the non standardness of open source all these years." [original text]

It was in the iPad forum where we found the least amount of anthropomorphic notions $(1.2 \%$ to $7.3 \%$ in the three different topics). Similar to Roomba conversations, the relation topic contained the most anthropomorphism (Fig. 3).

In the AIBO forum, $69.9 \%$ of the segments were about technical aspects (Fig. 2). Further, in $10.7 \%$ of the conversations, people described what they used their robotic dog for and in a relatively high proportion $(19.4 \%)$ people directly related to AIBO or described their feelings towards the device. In the following an example for a segment about rela-

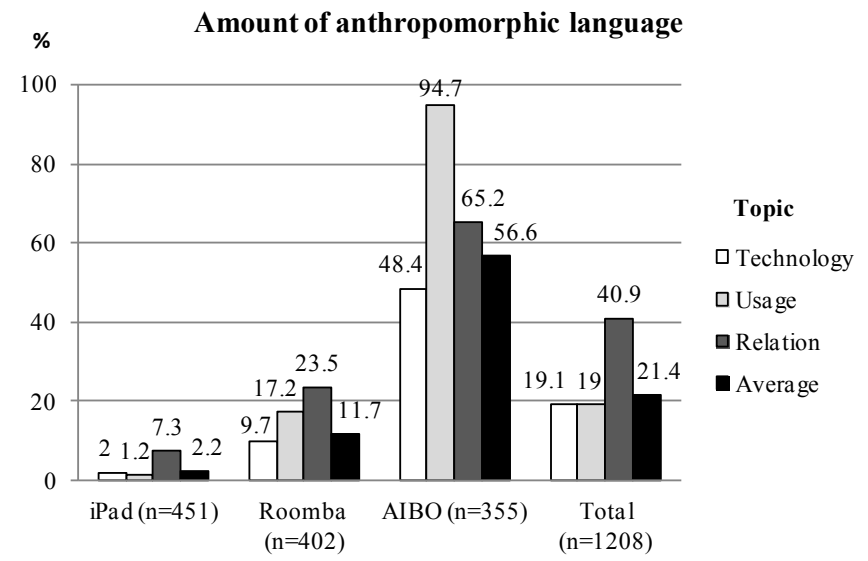

Fig. 3. Anthropomorphic language across three topics comparing three devices and in total tion containing also an anthropomorphic notion:

"Angus' [the AIBO's name] b'day is today. He's had a good day had plenty of dancing, talking and just being a superstar. " [original text]

As expected, people anthropomorphized AIBO much more than the other two devices. In between $48.4 \%$ and $94.7 \%$ of the postings in the three topic categories contained an anthropomorphic notion. Surprisingly, the highest amount was found in the usage category (Fig. 3).

Already from reading through the forums, it overall became clear that authors used drastically different languages to describe their devices. Especially the amount of notions of body-likeness (referring to parts of the device as if it would have a body) and postings where people named their device varied noteworthy. A statistical analysis was carried out to see whether the observed differences were due to the device itself or affected by the topic of the post, respectively.

\section{A. Anthropomorphism and the Device}

Hypothesized that due to the particular design of each of the devices, the AIBO forum would contain more anthropomorphisms than the posts in the Roomba and iPad forum $\left(\mathrm{H}_{1}\right)$, we applied a between subjects analysis. Statistically, a Chi-square test (Table 1) indicated a significant difference for the use of anthropomorphisms across the three devices $\left(\chi^{2}(\mathrm{~N}=1208,2)=383.5, \mathrm{p}<0.001\right.$; Table 1). Overall, $56.6 \%$ of all AIBO segments contained a notion of anthropomorphism, in comparison to $11.7 \%$ of the Roomba and $2.2 \%$ of the iPad segments (Fig. 3). This immense difference was also qualitatively prevalent when reading through the forum conversations. It further seems that on a subjective scale of life-likeness, Roomba is closer to the iPad than to AIBO, at least in matters of how they are described with words. This was against our presumption of AIBO and Roomba both being autonomous robots in contrast to the iPad being a static (but mobile) tablet computer. The values for the standardized residuals (Table 1) confirm this conclusion. This is an important result. It suggests that the relationship that users have to Roomba is more similar to a tool such as iPad than to the agent-like AIBO, despite the fact that, in itself, Roomba is closer to the latter one. Contrary, literature reports anecdotes where Roomba owners anthropomorphize

Table 1. Pearson Chi-Square Tests for Device And ANTHROPOMORPHISM

\begin{tabular}{cccc}
\hline \hline & $\begin{array}{c}\text { iPad } \\
(\mathrm{n}=451)\end{array}$ & $\begin{array}{c}\text { Roomba } \\
(\mathrm{n}=402)\end{array}$ & $\begin{array}{c}\text { AIBO } \\
(\mathrm{n}=355)\end{array}$ \\
\hline Chi-square & 31.40 & 6.68 & 5.67 \\
$\mathrm{df}$ & 2 & 2 & 2 \\
Sig. & $<.001$ & .035 & .059 \\
Std. Residual & -8.8 & -4.2 & 14.4 \\
\hline \hline Device*Anthrop $\left(\mathrm{x}^{2}(\mathrm{~N}=1208,2)=383.54, \mathrm{P}<0.001\right)$
\end{tabular}

Device*Anthrop $\left(\mathrm{X}^{2}(\mathrm{~N}=1208,2)=383.54, \mathrm{P}<0.001\right)$ 
their robot [18]. We interpret that either these are just rare examples or that those people who anthropomorphize their Roomba did not post in the forum we analyzed. As well in a longitudinal study carried out by the authors, most Roomba owners did not describe their robot in anthropomorphic ways. Along with novelty effects wearing off, social language towards the robot progressively faded out as it became more and more an everyday tool.

In contrast to the Roomba forum, authors in the AIBO forum more frequently named their robotic dog or attributed feelings and intentions to it, for instance:

“My ERS-111, Edgar, is also earless but it doesn't get him down [...]”. [original text]

Only very few authors named their Roomba and no one gave neither a name to their iPad nor attributed it with human-like characteristics. The few anthropomorphic notions in the iPad conversations were almost all about that people described the device as if it would actively or intentionally "decide" while carrying out an operation:

"Anyone that has ever accidently let itunes organize their library knows that this option should NEVER exist. Man what a mess it makes." [original text]

In accordance with a previous content analysis of an online forum about AIBO [17], people wrote about their "robotic puppies" using a variety of anthropomorphic expressions. However, this was hardly the case for both posts in the Roomba and iPad forums.

\section{B. Anthropomorphism and the Topic of the Post}

Our second assumption $\left(\mathrm{H}_{2}\right)$ was that across the three devices, segments describing the author's attitude or feeling towards the device (relation) would contain more anthropomorphism than when writing about technical aspects (technology) or how the product is used (usage). A Chi-squared analysis (Table 2) indicates significance between anthropomorphism and the topic of the segment $\left(\chi^{2}(\mathrm{~N}=1208,2)=\right.$ $32.4, \mathrm{p}<0.001)$. Further, the standardized residual rank scores suggest anthropomorphizing happens more in the relation category. However, qualitatively, this finding does not seem to hold for the AIBO forum. Whereas a descriptive analysis shows Roomba and iPad segments with topics technology and usage contain less anthropomorphism than those

TABle 2. PeARson Chi-SQuare Tests For TOPIC AND ANTHROPOMORPHISM

\begin{tabular}{cccc}
\hline \hline & $\begin{array}{c}\text { Technology } \\
(\mathrm{n}=818)\end{array}$ & $\begin{array}{c}\text { Usage } \\
(\mathrm{n}=263)\end{array}$ & $\begin{array}{c}\text { Relation } \\
(\mathrm{n}=127)\end{array}$ \\
\hline Chi-square & 203.50 & 174.54 & 38.11 \\
$\mathrm{df}$ & 2 & 2 & 2 \\
Sig. & $<.001$ & $<.001$ & $<.001$ \\
Std. Residual & -1.4 & -0.8 & 4.8 \\
\hline \hline Content*Anthrop $(\chi 2$ & $(\mathrm{N}=1208,2)=32.42, \mathrm{p}<0.001)$
\end{tabular}

about relation, this is not true for AIBO posts, where the amount of anthropomorphic language is highest for usage. We interpret that in contrast to the simple design (in terms of physical shape and interaction modalities), the animal-like embodiment of AIBO along with its ability to respond intelligently, encourages people to anthropomorphize it regardless of the topic of conversation. Especially the purpose of using a robotic dog is very different from using Roomba or the iPad. Whereas latter two have a clear purpose fulfilling a certain task, using AIBO does not have a clear purpose; it is more to play with it, for fun or entertainment. Indeed, activities carried out with AIBO can be considered as being playful and more social than letting Roomba vacuum one's floors or typing e-mails on the iPad while traveling. We interpret that this difference in how people use the device is reflected by the amount of anthropomorphic language in the usage category. This could mean that besides the shape of an artifact and its particular device, also the way how people use it seems to impact to which extent it is anthropomorphized.

\section{DISCUSSION}

The question that underlay our content analysis was what characteristics of an artifact encourage people to refer to it using anthropomorphic language, not taking into account psychological or cognitive factors.

We tackled this question by carrying out a content analysis of online forums which has already been shown to be a powerful approach [17]. Our study exceeds previous work by comparing three different devices as well as considering the topic of conversation in the forum. However, we do not want to over interpret our data. There are various drawbacks that limit the interpretability of our findings. On the one hand, we did not take into account factors external to the posts that could possibly impact the outcome, such as author or forum characteristics, the length of the postings, etc. For instance, it is possible, that each of the specific forum communities maintains a particular "community-language" which might in the first place imply a more frequent use of anthropomorphisms. On the other hand, it remains to investigate the anthropomorphic character of the human-like notions. More concretely, the question is how far people do not truly anthropomorphize based on the underlying social phenomenon but simply refer to the object's physical construction, such as AIBO's "ears". In fact, when talking about AIBO's ears, this might not be considered as a true anthropomorphic reference but rather as taking advantage of the fact that (parts of) the robot's physical shape imitate something else. Then in turn, the device might not appear to the human in a social way.

However, an interesting observation from our analysis is that anthropomorphic notions also seem to be related to how an artifact is used. This is concerned with the interaction and functionality an object supports but goes beyond. We interpret that in matters of anthropomorphism, not only the physical shape of a product but also the interaction it enables, its functionality as well as the way it is used, play a role. 
But how is AIBO different from Roomba and the iPad that people tend to anthropomorphize it much more? As mentioned in the findings section, our data suggests that the gap is bigger between Roomba and AIBO than between Roomba and the iPad. We have been surprised that only very few authors talked about their Roomba using anthropomorphic terms, such as by giving it a name. It has been described that owners of robotic vacuum cleaners tend to do so [18] [19]. Whereas in literature interactions with Roomba are described as being of relative social nature [18], our content analysis suggests that people hardly refer to it using anthropomorphic language. However, language is only a little part of the relation to an artifact and many other factors need to be taken into account. But still, what makes the huge difference then between Roomba and AIBO? Both are autonomously working robotic devices. We speculate that one big difference lies in the way that both devices are used as well as the purpose of interacting with them. Whereas Roomba works best while people are not present (low degree of interaction) and fulfills a specific task (vacuuming the floors), AIBO is meant to serve as a companion for people and actively encourages interaction. Its purpose is fairly unclear. In addition to that, AIBO responds to its user in an intelligent way and displays a somehow unpredictable behavior that can even make people surprise. This is quite different for Roomba and the iPad. We believe that a certain degree of unpredictability (and probably also failure) makes the robot to appear more humanlike and in turn facilitates a social relation.

\section{CONCLUSIONS}

Overall, we learnt that the phenomenon of anthropomorphism seems to be more difficult to grasp than we expected and is in fact a complex notion. This has already been pointed out by others [5]. Already the establishment of a robust coding scheme for anthropomorphic notions has been challenging and we are motivated to refine the proposed factors of anthropomorphic notions for future work.

We also found that the use of the term "anthropomorphism" can be controversial depending on context and background of the researchers. Anthropomorphism is discussed in a huge range of disciplines, such as in psychology, cognition, linguistics, philosophy, design, and robotics respectively.

In conclusion, the content analysis of online forum posts also helped us to get ideas about for what people use their devices for, which difficulties they encounter, technical constraints, as well as what they (dis)like about them. In terms of how the devices have been described, it was evident that $\mathrm{iPad}$ as well as Roomba were described as pure technologies / tools with a specific practical use or function. There have been only some (very) few anthropomorphic notions, whereas AIBO was described rather as a (robotic) pet with which people physically interact and that holds a personal value.

For the case of Roomba, this finding is confirmed by a longitudinal study the authors carried out in Swiss households. Results (will be published elsewhere) showed that on the long run, Roomba was not perceived as a robot and did not serve as a social agent despite in the very beginning and some anecdotic evidence of the opposite. People hardly anthropomorphized their vacuum cleaning robot.

\section{REFERENCES}

[1] N. Epley, A. Waytz, and J. T. Cacioppo, "On seeing human: A threefactor theory of anthropomorphism.," Psychological Review, vol. 114, pp. 864-886, 2007.

[2] L. R. Caporael, "Anthropomorphism and mechanomorphism: Two faces of the human machine," Computers in Human Behavior, vol. 2, no. 3, pp. 215-234, 1986.

[3] B. Reeves and C. Nass, The Media Equation: How People Treat Computers, Television, and New Media Like Real People and Places. Cambridge University Press, 1996.

[4] V. Venkatesh and F. D. Davis, "A Theoretical Extension of the Technology Acceptance Model: Four Longitudinal Field Studies," Management Science, vol. 46, no. 2, pp. 186-204, 2000.

[5] B. R. Duffy, "Anthropomorphism and the social robot," Robotics and Autonomous Systems, vol. 42, no. 3-4, pp. 177-190, Mar. 2003.

[6] F. Eyssel, F. Hegel, G. Horstmann, and C. Wagner, "Anthropomorphic inferences from emotional nonverbal cues: A case study," in 2010 IEEE RO-MAN, 2010, pp. 646-651.

[7] C. Breazeal, "Emotion and sociable humanoid robots," International Journal of Human-Computer Studies, vol. 59, no. 1-2, pp. 119-155, Jul. 2003.

[8] M. Coeckelbergh, "Humans, Animals, and Robots: A Phenomenological Approach to Human-Robot Relations," Int J of Soc Robotics, vol. 3, no. 2, pp. 197-204, Sep. 2010.

[9] J. Goetz and S. Kiesler, "Cooperation with a robotic assistant," in CHI '02 extended abstracts on Human factors in computing systems, New York, NY, USA, 2002, pp. 578-579.

[10] L. Axelrod and K. Hone, "E-motional advantage: performance and satisfaction gains with affective computing," in CHI '05 extended abstracts on Human factors in computing systems, New York, NY, USA, 2005, pp. 1192-1195.

[11] L. D. Riek, T.-C. Rabinowitch, B. Chakrabarti, and P. Robinson, "How anthropomorphism affects empathy toward robots," in Proceedings of the 4th ACM/IEEE international conference on Human robot interaction, New York, NY, USA, 2009, pp. 245-246.

[12] J. Goetz, S. Kiesler, and A. Powers, "Matching robot appearance and behavior to tasks to improve human-robot cooperation," in The 12th IEEE International Workshop on Robot and Human Interactive Communication, 2003. Proceedings. ROMAN 2003, 2003, pp. 55- 60.

[13] S. Kiesler and J. Goetz, "Mental models of robotic assistants," in CHI ' 02 extended abstracts on Human factors in computing systems, Minneapolis, Minnesota, USA, 2002, pp. 576-577.

[14] S. Lee, I. Y. Lau, S. Kiesler, and C.-Y. Chiu, "Human Mental Models of Humanoid Robots," in Proceedings of the 2005 IEEE International Conference on Robotics and Automation, 2005. ICRA 2005, 2005, pp. 2767- 2772 .

[15] S. Krach, F. Hegel, B. Wrede, G. Sagerer, F. Binkofski, and T. Kircher, "Can Machines Think? Interaction and Perspective Taking with Robots Investigated via fMRI," PLoS ONE, vol. 3, no. 7, p. e2597, Jul. 2008.

[16] C. Bartneck, D. Kulić, E. Croft, and S. Zoghbi, "Measurement Instruments for the Anthropomorphism, Animacy, Likeability, Perceived Intelligence, and Perceived Safety of Robots," International Journal of Social Robotics, vol. 1, no. 1, pp. 71-81, Nov. 2008.

[17] B. Friedman, P. H. Kahn,Jr., and J. Hagman, "Hardware companions?: what online AIBO discussion forums reveal about the humanrobotic relationship," in Proceedings of the SIGCHI conference on Human factors in computing systems, New York, NY, USA, 2003, pp. $273-280$.

[18] J. Forlizzi, "How robotic products become social products: an ethnographic study of cleaning in the home," in Proceedings of the ACM/IEEE international conference on Human-robot interaction, New York, NY, USA, 2007, pp. 129-136.

[19] J. Sung, R. E. Grinter, and H. I. Christensen, "Domestic Robot Ecology," Int J of Soc Robotics, vol. 2, no. 4, pp. 417-429, Jul. 2010. 